\title{
Utilization of Mercaptoethylgluconamide for Depleting Human Cystinotic Fibroblasts of their Accumulated Lysosomal Cystine
}

\author{
RONALD L. PISONI, KAREN M. LISOWSKI, ROSEMARY M. LEMONS, AND JESS G. THOENE \\ Department of Pediatrics and Communicable Diseases, The University of Michigan School of Medicine, \\ Ann Arbor, Michigan 48109-2029
}

\begin{abstract}
Human cystinotic fibroblasts were completely depleted of their accumulated intracellular free cystine within a 2 -h time interval when exposed to culture medium containing between 1 and $5 \mathrm{mM}$ mercaptoethylgluconamide. This cystine-depleting action of mercaptoethylgluconamide was observed with three different human cystinotic fibroblast cell lines and with all three cell lines, $2 \mathrm{mM}$ mercaptoethylgluconamide was as effective as 1 $\mathrm{mM}$ cysteamine in depleting cells of their intracellular free cystine. Cell viability was excellent for cystinotic fibroblasts exposed to $2 \mathrm{mM}$ mercaptoethylgluconamide for up to 6 days in duration. Mercaptoethylgluconamide $(2 \mathrm{mM})$ was sufficiently stable under cell culture conditions such that a single addition of mercaptoethylgluconamide maintained cystine depletion in human cystinotic fibroblasts for at least a 4-day period. In contrast to cysteamine, $2 \mathrm{mM}$ mercaptoethylgluconamide was not capable of depleting the cystine content of isolated cystinotic lysosomes, implying that cellular integrity is necessary to achieve cystine depletion by mercaptoethylgluconamide. The efficient cystine-depleting action of mercaptoethylgluconamide coupled with its lack of offensive odor encourage further investigation of this agent to possibly complement or supplant the use of cysteamine in the treatment of nephropathic cystinosis. (Pediatr Res 26: 73-76, 1989)
\end{abstract}

\section{Abbreviations}

MEG, mercaptoethylgluconamide

MOPS, 3-( $(N$-morpholino $)$ propane sulfonic acid $)$

NEM, $N$-ethylmaleimide

\footnotetext{
Nephropathic cystinosis is an autosomal recessively inherited disease characterized by a high intralysosomal accumulation of the amino acid, cystine, which results from a defect in lysosomal cystine transport (1-4). Early clinical manifestations of this disease apparent by 12-18 mo of age include the renal Fanconi syndrome, photophobia, retinopathy, keratopathy, and growth retardation (1). Intracellular cystine levels are elevated to different degrees in different tissues, ranging from 10- to 100-fold higher than the normal range in polymorphonuclear leukocytes, fibroblasts, Kupffer cells, and cells of the kidney, rectal mucosa, bone marrow, lymph nodes, thyroid, muscle, and conjunctiva $(1,5-7)$. Glomerular failure eventually occurs in nephropathic cystinosis by the age of 10 . In 1976, Thoene et al. (8) demon-

Received December 27, 1988; accepted March 16, 1989

Correspondence Dr. Ronald L. Pisoni, 2618 SPH I, Department of Pediatrics and Communicable Diseases, University of Michigan, Ann Arbor, MI 48109-2029.

Supported by Grant DK25548 from the National Institutes of Health.
}

strated that the aminothiol, cysteamine, could effectively deplete cystinotic cells of their high intralysosomal accumulation of cystine both in vitro and in vivo. A subsequent multicenter trial has shown that cysteamine treatment preserves renal glomerular function and improves linear growth (9). Despite the clinical success of cysteamine it has a significant disadvantage in that it has an unpalatable taste, hence other cystine-depleting drugs are required. This report describes the ability of the carbohydrate derivative, MEG, to deplete human cystinotic fibroblasts of their accumulated cystine in a very effective manner.

\section{MATERIALS AND METHODS}

Diploid human fibroblasts were cultured in Coon's modified Ham's F-12 medium supplemented with $10 \%$ (v/v) fetal bovine serum and incubated at $37^{\circ} \mathrm{C}$ in a humidified incubator flushed with an air $/ \mathrm{CO}_{2}$ (19:1) mixture. Cystine-free medium was the same medium with cystine deleted and supplemented with $10 \%$ dialyzed fetal bovine serum. Experiments were performed with fibroblasts grown to a confluent state on $60-\mathrm{mm}$ culture dishes. WG1522 is a fibroblast line derived from skin biopsy of a 11 mo-old cystinotic child, GM0090A is a human cystinotic fetal lung fibroblast cell line purchased from the Human Genetic Mutant Cell Repository (Camden, NJ), and DFL is a human fetal lung fibroblast line grown from a cystinotic fetus.

Cells were harvested by trypsin treatment and cell number determined by a model $Z_{F}$ Coulter counter (Coulter Instruments, Inc., Hialeah, FL). Cell viabilities were determined by trypan blue exclusion (11) and by a 4-h cell attachment assay. In the cell attachment assay, trypsinized cells were allowed to attach to a $60-\mathrm{mm}$ culture dish for $4 \mathrm{~h}$ at $37^{\circ} \mathrm{C}$ in culture medium containing $10 \%$ fetal bovine serum. Then each dish was washed three times with PBS, retrypsinized, and the number of cells recovered at this point divided by the number of cells at the initial trypsinization was taken as a more stringent measure of cell viability (12).

Total free cystine was measured by a cystine binding protein assay (13). In other experiments, confluent fibroblast monolayers were cultured for $18-24 \mathrm{~h}$ in complete medium containing ${ }^{35} \mathrm{~S}$ cystine $\left(1.0 \times 10^{6} \mathrm{cpm} / \mathrm{ml}\right)$ to radiolabel the lysosomal cystine pool. Cell monolayers were then washed three times with PBS, and incubated in cystine-free culture medium containing either $1 \mathrm{mM}$ cysteamine, $2 \mathrm{mM} \mathrm{MEG}$, or, in the case of the control, no further addition. At intervals, the cells were harvested by trypsin treatment and immediately broken by sonic disruption in a $5 \mathrm{mM}$ N-ethylmaleimide/ $100 \mathrm{mM}$ sodium phosphate buffer, $\mathrm{pH}$ 7.4. Cell proteins were precipitated by centrifugation after addition of sulfosalicylic acid to $3 \%(\mathrm{v} / \mathrm{v})$, and the supernatant, along with internal standards of cystine and cysteine-NEM, were analyzed by one-dimensional high voltage electrophoresis in $6 \%$ formic acid at $3500 \mathrm{~V}$ for $25 \mathrm{~min}$. The paper was dried, the 
standards visualized by the use of a ninhydrin spray, and radioactivity comigrating with the cystine standard was cut out and counted in $10 \mathrm{ml}$ of Bio-Safe II scintillation fluid (Research Products International Corp., Mt. Prospect, IL) (8).

Purified fibroblast lysosomes were prepared as previously described by centrifugation through Percoll density gradients (14). These lysosomes were pre-loaded with ${ }^{35} \mathrm{~S}$-cystine by incubating for $1 \mathrm{~h}$ at $37^{\circ} \mathrm{C}$ with $10 \mu \mathrm{Ci}$ of ${ }^{35} \mathrm{~S}$-cystine dimethyl ester $(2 \mathrm{Ci} /$ $\mathrm{mmol}$ ) in $0.3 \mathrm{ml}$ of $10 \mathrm{mM}$ MOPS/Tris pH 7.6 buffer containing $0.25 \mathrm{M}$ sucrose and $1 \mathrm{mM} \mathrm{Na} \mathrm{EDTA}_{2}$ (15). Lysosomes were then diluted to $1.5 \mathrm{ml}$ with ice-cold buffer, centrifuged for $8 \mathrm{~min}$ at $4^{\circ} \mathrm{C}$ in a model 5415 Eppendorf microcentrifuge (Brinkmann Instruments, Westbury, NY), and the supernatant discarded. The lysosomal pellet was resuspended in $1.5 \mathrm{ml}$ of ice-cold buffer and washed once more by centrifugation. The final lysosomal pellet was resuspended to $115 \mu \mathrm{l}$ with ice-cold $40 \mathrm{mM}$ MOPS/ Tris pH 7.0 buffer containing $0.25 \mathrm{M}$ sucrose. The ${ }^{35} \mathrm{~S}$-cystineloaded lysosomal suspension was divided in equal aliquots and then mixed with an equivalent volume of $40 \mathrm{mM}$ MOPS/Tris pH 7.0 buffer containing either $2 \mathrm{mM}$ cysteamine, $4 \mathrm{mM}$ MEG or no further addition. The suspensions were incubated at $37^{\circ} \mathrm{C}$ for $45 \mathrm{~min}$ then diluted to $1.5 \mathrm{ml}$ with ice-cold $20 \mathrm{mM}$ MOPS/ Tris pH 7.0 buffer containing $0.275 \mathrm{M}$ mannitol and $10 \mathrm{mM} \mathrm{N}$ ethylmaleimide and centrifuged for $10 \mathrm{~min}$ at $15,600 \times g$ in an Eppendorf microcentrifuge at $4^{\circ} \mathrm{C}$. The supernatants were discarded and the lysosomal pellets were resuspended in $90 \mu \mathrm{l}$ of 12 $\mathrm{mM}$ N-ethylmaleimide in $10 \mathrm{mM}$ sodium phosphate $\mathrm{pH} 7.5$ buffer containing $0.1 \%$ Triton. Proteins were precipitated in each tube by centrifugation after the addition of sulfosalicyclic acid to $4 \%(\mathrm{v} / \mathrm{v})$ and $10 \mu \mathrm{l}$ aliquots of the supernatants were analyzed by one-dimensional high voltage electrophoresis as described above. The ${ }^{35} \mathrm{~S}$-cystine dimethyl ester was prepared from ${ }^{35} \mathrm{~S}$ cystine as described previously (16) and purified by methanol elution of the radioactivity migrating with the cystine dimethyl ester standard after one-dimensional high voltage electrophoresis in $6 \%$ formic acid.

Cysteamine was purchased from Sigma Chemical Co. (St. Louis, MO), MEG from Vega Biotechnologies', Inc. (Tucson, AZ) ${ }^{35} \mathrm{~S}$-L-cystine $(>300 \mathrm{Ci} / \mathrm{mmol})$ from Dupont/New England Nuclear (Boston, MA) and fetal bovine serum and Coon's modified Ham's F-12 medium were from GIBCO, Grand Island, NY.

\section{RESULTS}

We have previously shown that when cystinotic fibroblasts are placed in culture medium containing $1 \mathrm{mM}$ cysteamine, the cystine content of these fibroblasts is rapidly depleted, reaching within 30-60 min, a level approaching that found in normal fibroblasts (8). We now report that $2 \mathrm{mM}$ MEG depletes cystinotic fibroblasts of their intracellular free cystine to the same low level as achieved with $1 \mathrm{mM}$ cysteamine. Time courses comparing the cystine depleting action of $1 \mathrm{mM}$ cysteamine and $2 \mathrm{mM}$ MEG are shown in Figure 1. Nearly identical time courses of cystine depletion by $2 \mathrm{mM}$ MEG were obtained with three different cystinotic fibroblast cell lines. Time courses of cystine depletion were very similar whether intracellular cystine levels were quantified using a cystine binding protein assay or by measurement of free $\left.{ }^{35} \mathrm{~S}\right]$ cystine as described in "Materials and Methods" (data not shown). In all cystinotic cell lines, the rate of cystine depletion by $2 \mathrm{mM}$ MEG was somewhat slower than that by cysteamine, requiring approximately $2 \mathrm{~h}$ to reach complete depletion as compared to $30 \mathrm{~min}$ for $1 \mathrm{mM}$ cysteamine. For reference, the structures of MEG and cysteamine are provided in Figure 2.

A study of the dependence of cystine depletion on the concentration of mercaptoethylgluconamide indicates that MEG concentrations as low as $0.1 \mathrm{mM}$ provide a substantial level of

'MEG is no longer routinely available from Vega but a method for its synthesis is provided in Reference 10.

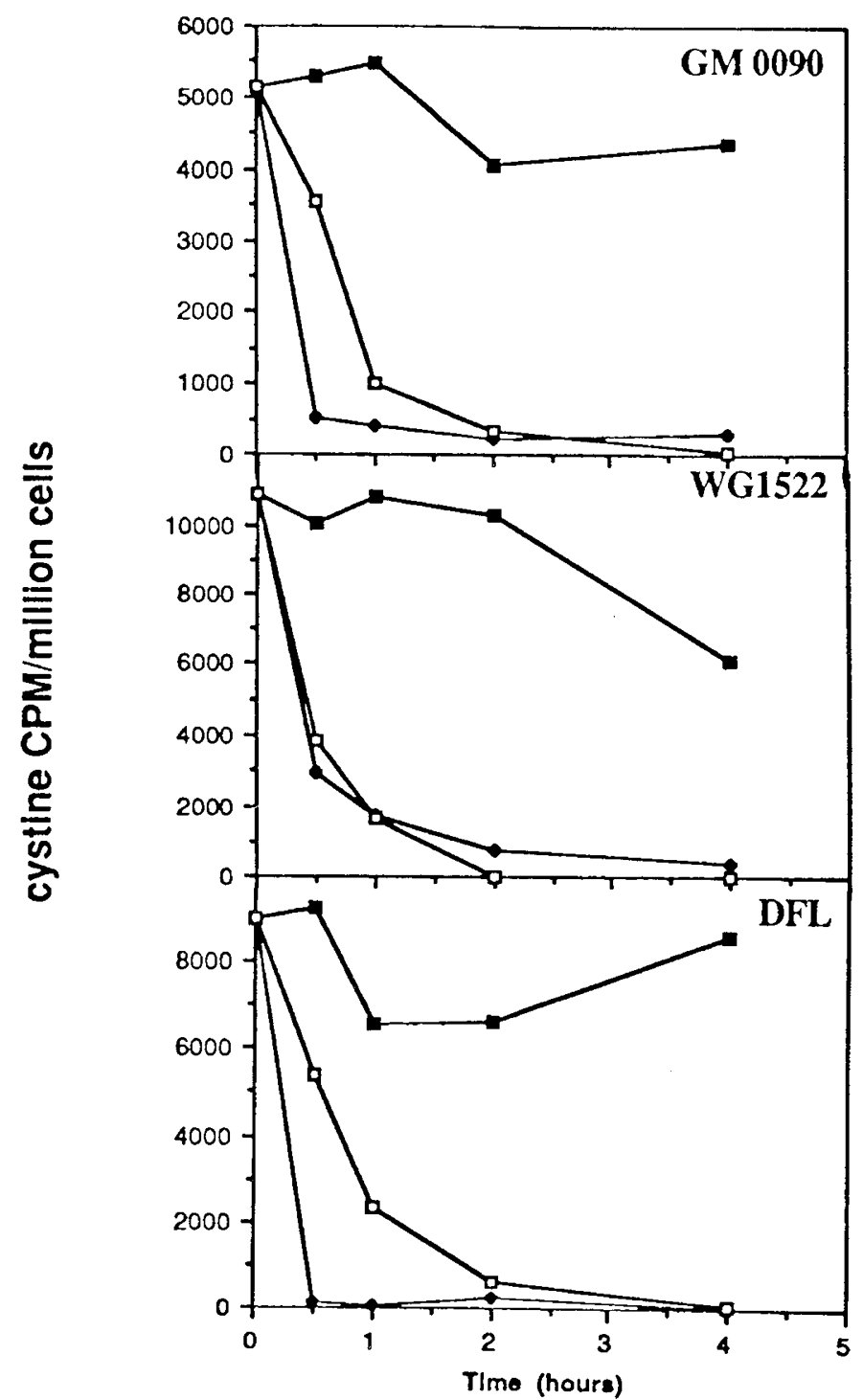

Fig. 1. Comparison of the ability of mercaptoethylgluconamide and cysteamine to deplete the pre-labeled ${ }^{35} \mathrm{~S}$-cystine pool of three different cystinotic cell lines. Confluent monolayers of each cystinotic cell line were radioactively labeled with ${ }^{35} \mathrm{~S}$-L-cystine for $18 \mathrm{~h}$. Monolayers were then washed three times with PBS and at time zero placed in cystinefree culture media containing either $2 \mathrm{mM}$, mercaptoethylgluconamide $(\square), 1 \mathrm{mM}$ cysteamine $(\diamond)$, or no further addition in the case of the control (a). At the indicated times, plates were harvested and cell numbers and the amount of free ${ }^{35}$ S-cystine within the fibroblasts was determined as described in "Materials and Methods." The cell lines used were, upper panel: GM 0090A, middle panel: WG 1522, and lower panel: DFL.

cystine depletion within $4 \mathrm{~h}$ (Fig. 3). Increasing the concentration of mercaptoethylgluconamide results in a faster rate of cystine depletion although the same final level of depletion is reached with concentrations of MEG ranging from 1 to $5 \mathrm{mM}$. The rate of cystine depletion obtained with $5 \mathrm{mM}$ MEG is approximately one-half the rate obtained with $1 \mathrm{mM}$ cysteamine shown in Figure 1.

Several experiments were performed to assess the effects of exposing cystinotic fibroblasts to $2 \mathrm{mM}$ MEG for periods of time more than $4 \mathrm{~h}$. Culture dishes of cystinotic fibroblasts were placed in medium containing $2 \mathrm{mM}$ MEG on day 0 , the cells were subsequently harvested at days $0,1,2,3$, and 4 of culture, and intracellular free cystine levels were measured. The results shown in Figure 4 indicate that MEG is able to maintain cystine 


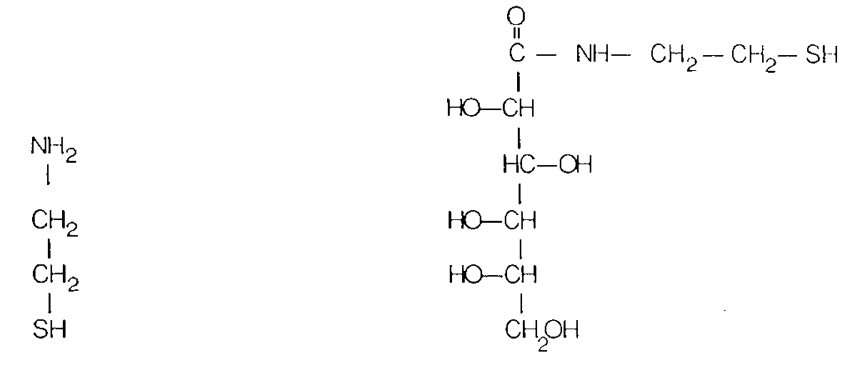

Cysteamine

Mercaptoethylgluconamide

Fig. 2. Structural comparison of mercaptoethylgluconamide and cysteamine.

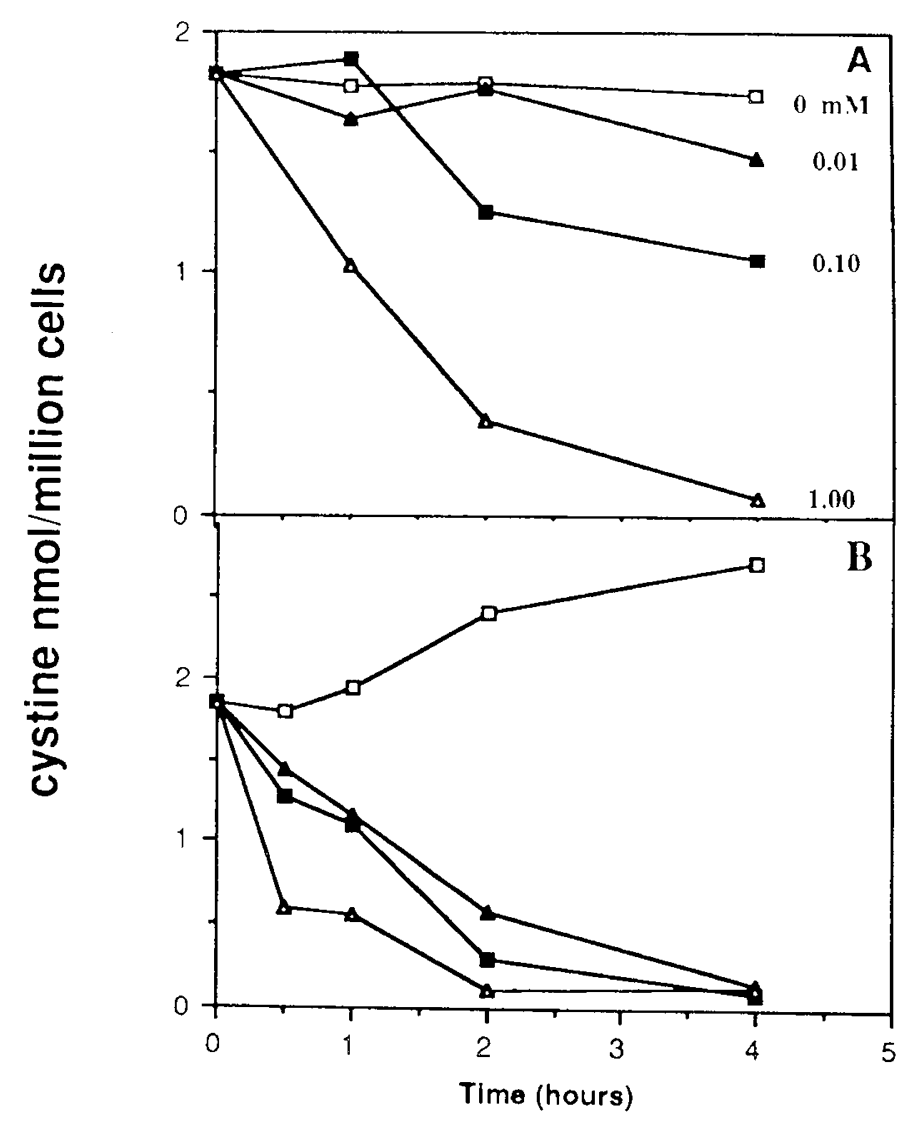

Fig. 3. The effect of mercaptoethylgluconamide concentration on the cystine content of cystinotic fibroblasts. Plates of confluent GM 0090A cystinotic fibroblasts were washed three times with PBS and at time zero were placed in cystine-free culture media containing mercaptoethylgluconamide of the indicated concentration. Cells were harvested by trypsin treatment at the times indicated and the intracellular nonprotein cystine content was measured by means of the cystine binding assay. Cell number was measured with a Coulter counter. $A, M E G$ concentration from $0-1$ $\mathrm{mM} ; B$, MEG concentrations, $0 \mathrm{mM}(\square), 1 \mathrm{mM}(\mathbf{\Lambda}), 2 \mathrm{mM}(\boldsymbol{\square})$, and 5 $\mathrm{mM}(\triangle)$.

depletion of cystinotic fibroblasts over the entire 4-day period after the initial addition of MEG. Again, the level of cystine depletion obtained with MEG is identical to that achieved with cysteamine. Thus, $2 \mathrm{mM}$ MEG is sufficiently stable under standard cell culture conditions so as to maintain a cystine-depleted state in human cystinotic fibroblasts over a period of at least several days.

To assess the possible adverse effects of $M E G$ on cell viability, cystinotic fibroblasts were cultured in complete medium containing $2 \mathrm{mM}$ MEG for 2 days, the culture medium was then replaced with fresh medium containing $2 \mathrm{mM}$ MEG and cultured for an

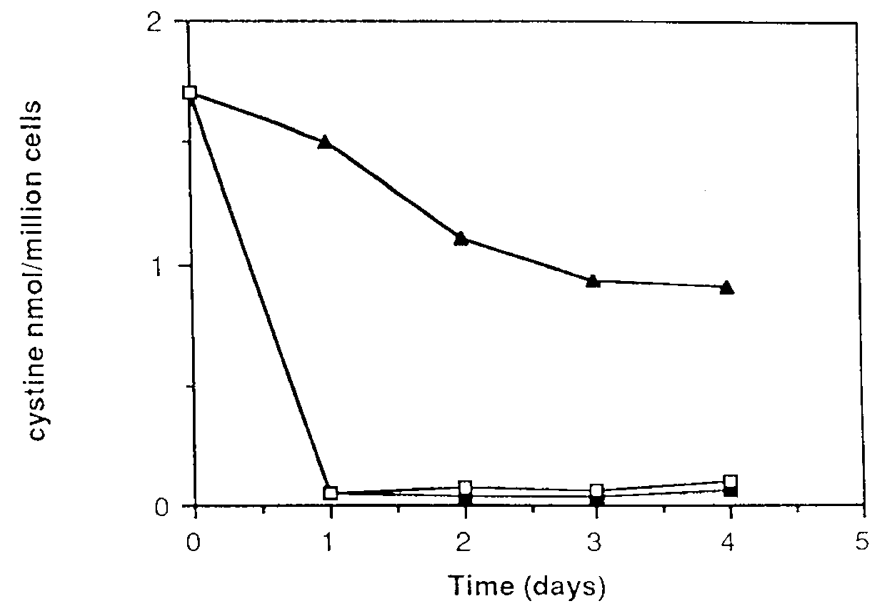

Fig. 4. The duration of the cystine depletion effect of $2 \mathrm{mM}$ mercaptoethylgluconamide during 4 days in culture. Confluent monolayers of GM 0090A cystinotic fibroblasts were washed three times with PBS and at time zero were placed in complete media containing either $2 \mathrm{mM}$ mercaptoethylgluconamide $(\square), 1 \mathrm{mM}$ cysteamine $(\boldsymbol{\square})$, or as a control, no further addition $(\mathbf{\Lambda})$. Each plate was cultured for the indicated period of time without changing the media and with no further addition of MEG or cysteamine. Cell monolayers were then harvested at their respective time points by trypsin treatment and the intracellular nonprotein cystine content was measured by the cystine binding assay. Cell numbers were measured with a Coulter counter.

additional 4 days. The cystinotic fibroblasts when observed with a light microscope each day during this period appeared as healthy as cystinotic fibroblasts not exposed to MEG. Cell viability at the end of the 6-day exposure period was excellent for the MEG-treated fibroblasts, displaying $90 \%$ exclusion of trypan blue and $58 \%$ cell attachment in a 4 -h cell attachment assay (data not shown). Control cells, not exposed to MEG, show $51 \%$ attachment (12). Cell viability was also retained if the MEGcontaining media was changed every day during a 4-day period. Throughout this 4-day period, $2 \mathrm{mM}$ MEG kept intracellular free cystine levels depleted to a low level equivalent to that obtained with $1 \mathrm{mM}$ cysteamine (data not shown).

We have shown previously that the aminothiols, cysteamine, and thiocholine, are able to permeate the membranes of isolated fibroblast lysosomes and convert intralysosomal cystine to mixed disulfides which are then exported from the lysosome by the lysosomal system c transport system (15). When lysosomes preloaded with $\left[{ }^{35} \mathrm{~S}\right]$ cystine were incubated with $2 \mathrm{mM}$ MEG for 45 min at $37^{\circ} \mathrm{C}$, however, we did not observe any cystine depletion beyond that seen in the buffer control (Table 1), whereas cysteamine caused marked cystine depletion. This lack of effect by MEG on the cystine content of isolated lysosomes may indicate that processes dependent upon cellular integrity are necessary for delivery of the cystine-depleting activity of MEG to the lysosomal compartment. In a separate experiment in which $\left[{ }^{35} \mathrm{~S}\right]$ cystine was directly exposed to $2 \mathrm{mM}$ MEG in a $40 \mathrm{mM}$ MOPS/Tris $\mathrm{pH} 7.0$ buffer for $10 \mathrm{~min}$ at $21^{\circ} \mathrm{C}$ and then the reaction stopped by the addition of $10 \mathrm{mM} \mathrm{N}$-ethylmaleimide, all of the cystine was converted to a product(s) comigrating with the cysteine-NEM standard during high voltage electrophoresis (data not shown). Whether these product(s) are solely cysteine-NEM or a mixture of cysteine-NEM and the mixed disulfide of cysteine and MEG has not been determined.

\section{DISCUSSION}

We have found that the carbohydrate derivative, MEG, is capable of depleting human cystinotic fibroblasts of their cystine to the same low level as achieved with cysteamine. The basis of cysteamine therapy of nephropathic cystinosis rests on the ability 
Table 1. Ability of $2 \mathrm{mM}$ mercaptoethylgluconamide or $1 \mathrm{mM}$ cysteamine to deplete the cystine content of isolated fibroblast lysosomes*

\begin{tabular}{cc}
\hline & ${ }^{35}$ S-cystine, \\
Incubation condition & $\mathrm{cpm} / \mathrm{U}$ hexosaminidase \\
\hline
\end{tabular}

\begin{tabular}{|c|c|}
\hline $\begin{array}{l}{ }^{35} \text { S-cystine-loaded lysosomes at be- } \\
\text { ginning of } 45 \mathrm{~min} \text { incubation }(\mathrm{t}= \\
0 \mathrm{~min})\end{array}$ & 148 \\
\hline $\begin{array}{l}\text { Lysosomes + buffer after } 45 \mathrm{~min} \text { at } \\
37^{\circ} \mathrm{C}\end{array}$ & 118 \\
\hline $\begin{array}{l}\text { Lysosomes }+2 \mathrm{mM} \mathrm{MEG} \mathrm{after} 45 \\
\text { min at } 37^{\circ} \mathrm{C}\end{array}$ & 112 \\
\hline $\begin{array}{l}\text { Lysosomes }+1 \mathrm{mM} \text { cysteamine } \\
\text { after } 45 \mathrm{~min} \text { at } 37^{\circ} \mathrm{C}\end{array}$ & 30 \\
\hline
\end{tabular}

* Percoll-purified lysosomes prepared from three confluent roller bottles of GM 0090A cystinotic fibroblasts were loaded with ${ }^{35} \mathrm{~S}$-cystine by incubation with ${ }^{35}$ S-cystine dimethyl ester as described in "Materials and Methods." The ${ }^{35}$ S-cystine-loaded lysosomes were washed free of unaccumulated ${ }^{35} \mathrm{~S}$-cystine dimethyl ester by centrifugation and then incubated at $37^{\circ} \mathrm{C}$ for $45 \mathrm{~min}$ with $40 \mathrm{mM}$ MOPS/Tris pH 7.0 buffer containing either $1 \mathrm{mM}$ cysteamine, $2 \mathrm{mM}$ mercaptoethylgluconamide, or no further addition. At the end of this time interval, lysosomes were collected by centrifugation and the amount of free ${ }^{35} \mathrm{~S}$-cystine remaining in each lysosomal pellet was determined by high voltage electrophoresis as described in "Materials and Methods."

of cysteamine to react with lysosomal cystine by sulfhydryl/ disulfide exchange to form cysteine and the mixed disulfide of cysteamine and cysteine (8). The mixed disulfide of cysteamine and cysteine is then transported out of the lysosome by lysosomal system c $(15,17)$ whereas cysteine may leave the lysosome by the transport systems for the small neutral amino acids, systems $\mathrm{e}$ and $\mathrm{f}(14)$. Both reaction products then enter normal cellular metabolic pathways. Thus, cysteamine produces cystine depletion by converting cystine to products which are recognized by other functional lysosomal transport systems, allowing for net exodus of cystine. The mechanism responsible for the cystinedepleting action of MEG is not yet clear. When directly reacted with cystine, MEG can readily reduce cystine. Our experiments indicate, however, that MEG by itself is not capable of traversing the membranes of isolated lysosomes. This suggests that either an MEG metabolite, possibly formed in the cytosol, is responsible for the cystine-dêpleting action of MEG, or that cellular integrity is required for the delivery of MEG to the lysosomal compartment allowing reaction with intralysosomal cystine. A mixed disulfide of MEG and cysteine formed in the lysosomal compartment is not likely to be recognized by lysosomal system $\mathrm{c}$ because it would lack a positively charged side chain necessary for recognition by lysosomal system $\mathrm{c}$. The mixed disulfide of MEG and cysteine, however, could possibly be recognized by the transport system for large, hydrophobic amino acids, lysosomal system h (18), or by a transport system not yet characterized. Regarding the possible metabolism of MEG, it is conceivable that an enzymatic hydrolysis of the amide bond in MEG could produce cysteamine in situ which then could traverse the lysosomal membrane and cause cystine depletion.

MEG represents a new class of agent capable of depleting cystinotic fibroblasts of their accumulated cystine. Good retention of cell viability is observed when cystinotic fibroblasts are exposed to $2 \mathrm{mM}$ MEG for periods of at least 6 days in duration. Importantly, we have not noticed any substantial offensive odor associated with the MEG used in this study and others have recently noted that MEG is odorless (10). Thus, in this regard MEG appears superior to cysteamine which has a conspicuous thiol odor and taste.

MEG also displays good solubility and, as a carbohydrate, could be joined to other carbohydrates by specific linkages to yield a polymer containing many MEG residues. This provides new opportunities for drug design. For example, such a polymer with the appropriate linkages, cleavable by enzymes present in lacrimal secretions could permit a sustained release of MEG for dissolving the corneal crystals arising in cystinosis (19-22). The potential beneficial applications of mercaptoethylgluconamide for complementing or supplanting the use of cysteamine in treating cystinosis or possibly other diseases warrants further clinical investigation.

\section{REFERENCES}

1. Gahl W, Renlund M, Thoene J Lysosomal transport disorders-cystinosis and sialic acid storage disorders. In: Scriver C (ed) The Metabolic Basis of Inherited Disease. McGraw-Hill, New York, In press

2. Gahl W, Bashan N, Tietze F, Bernardini I, Schulman JD 1982 Cystine transport is defective in isolated leukocyte lysosomes from patients with cystinosis. Science 217:1263-1265

3. Gahl WA, Tietze F, Bashan N, Steinherz R, Schulman, JD 1982 Defective cystine exodus from isolated lysosome-rich fractions of cystinotic leukocytes. J Biol Chem 257:9570-9575

4. Jonas AJ, Smith ML, Schneider JA 1982 ATP-dependent lysosomal cystine efflux is defective in cystinosis. $\mathrm{J}$ Biol Chem 257:13185-13188

5. Gahl WA 1986 Cystinosis coming of age. Adv Pediatr 33:95-126

6. Thoene JG 1988 Clinical characteristics and recent progress in nephropathic cystinosis. In: Gahl WA (moderator) Cystinosis: Progress in a Prototypic Disease. Ann Intern Med 109:557-559

7. Gahl WA, Dalakas, MJC, Charnas L, Chen KTK, Pezeshkpour GH, Kuwabara T, Avis SL, Chesney RW, Fink J, Hutchison HT 1988 Myopathy and cystine storage in muscles in a patient with nephropathic cystinosis. N Engl J Med 319:1461-1464

8. Thoene JG, Oshima RG, Crawhall JC, Olson DL, Schneider JA 1976 Cystinosis: intracellular cystine depletion by aminothiols in vitro and in vivo. $\mathrm{J}$ Clin Invest 58:180-189

9. Gahl WA, Reed GF, Thoene JG, Schulman JD, Rizzo WB, Jonas AJ, Denman DW, Schlesselman JJ, Corden BJ, Schneider JA 1987 Cysteamine therapy for children with nephropathic cystinosis. N Engl J Med 316:971-977

10. Tsushima R, Kondo A, Mita K 1987 N-mercaptoalkygluconamide Chem Abstr 106:156824q

1. Freshney RI 1983 Quantitation and experimental design. In: Culture of Animal Cells. Alan R. Liss, Inc., New York, p. 209

12. Lemons R, Forster S, Thoene J 1988 Protein microinjection by protease permeabilization of fibroblasts Anal Biochem 172:219-227

13. Oshima RG, Willis, RC, Furlong, CE, Schneider, JA 1974 Binding assays for amino acids. The utilization of a cystine binding protein from Escherichia coli for the determination of acid-soluble cystine in small physiological samples. J Biol Chem 249:6033-6039

14. Pisoni RL, Flickinger KS, Thoene JG, Christensen HN. 1987 Characterization of carrier-mediated transport systems for small neutral amino acids in human ibroblast lysosomes. J Biol Chem 262:6010-6017

15. Pisoni, RL, Thoene JG, Lemons RM, Christensen, HN 1987 Important differences in cationic amino acids transport by lysosomal system $\mathrm{c}$ and system $\mathrm{y}^{+}$of the human fibroblast. J Biol Chem 262:15011-15018

16. Steinherz R, Tietze F, Raiford F, Gahl W, Schulman J 1982 Patterns of amino acid efflux from isolated normal and cystinotic human leukocyte lysosomes. Biol Chem 257:6041-6049

17. Pisoni R, Thoene $\mathbf{J}$, Christensen $\mathrm{H} 1985$ Detection and characterization of carrier-mediated cationic amino acid transport in lysosomes of normal and cystinotic human fibroblasts:role in therapeutic cystine removal. J Biol Chem 260:4791-4798

18. Bernar J, Tietze F, Kohn LD, Bernardini I, Harper GS, Grollman EF, Gahl WA 1986 Characteristics of a lysosomal membrane transport system for tyrosine and other neutral amino acids in rat thyroid cells $\mathrm{J}$ Biol Chem 261:17107-17112

19. Kaiser-Kupfer MI, Caruso RC, Minkler DS, Gahl WA 1986 Long-term ocular manifestations in nephropathic cystinosis. Arch Opthalmol 104:706-711

20. Cogan DG, Kuwabara T 1960 Ocular pathology of cystinosis. Arch Opthalmol 63:51-57

21. Francois J, Hanssens M, Coppieters R, Evens L 1972 Cystinosis: a clinical and histopathologic study. Am J Ophthalmol 73:643-650

22. Wong VG 1973 The eye and cystinosis. In: Schulman JD (ed) Cystinosis. United States Government Printing Office, Washington, DC, DHEW Publication no (NIH) 72-249, pp 23-35 